\title{
A Testbed System For Impact of QoS Parameter in Wireless ad Hoc Network
}

\author{
Ngo Hai Anh, Pham Thanh Giang
}

\begin{abstract}
In our paper, we evaluate the impact of some QoS parameters on multimedia data in IEEE 802.11 wireless networks by deploying an experimental testbed systems. The evaluation results show that Contention Window $(C W)$ value has a great influence on the throughput ratio between multimedia data types.
\end{abstract}

Keywords : Multimedia data, network performance, testbed, throughput, wireless ad hoc.

\section{INTRODUCTION}

To officially apply a standard in IEEE 802.11[1] it is necessary to undergo many testing and evaluation processes to prove its effectiveness. The evaluation of network parameters in general is difficult, especially with wireless networks due to the variety of hardware, software, network parameters and it is difficult to have an optimal method for evaluating all the parameters. Some of the main methods are usually implemented as modeling, simulation and emulation. The modeling method has the advantage that it is easy to add parameters, accurate results because it can be proved by mathematics, the model is very generalized; however, the disadvantage is that it is difficult to deploy because the actual network environment has many impacting factors. The simulation method has the advantage of low cost, the network parameters can be easily changed and observed by setting the value in simulation software; The disadvantage of this method is that it is difficult to prove exactly like the modeling method and the evaluation factors are nonetheless due to the simulation software still does not reach the same as the reality. The emulation method, or in other words, using real equipment to simulate a network environment that is almost realistic to evaluate the network experiments, this method has advantages over both modeling and simulation methods, similar to the real networks, but there are also difficulties due to the equipment investment cost, only simulating a part but not entire all the network (because there are many devices, many users). In wireless networks, the device-based network test environment (testbed) is increasingly used because the device costs have dropped significantly as well as there are many support tools (framework) for using and building a

Revised Manuscript Received on December 30, 2019.

* Correspondence Author

Ngo Hai Anh*, Institute of Information Technology, Vietnam Academy of Science and Technology. E-mail: ngohaianh@ioit.ac.vn

Pham Thanh Giang, Institute of Information Technology, Vietnam Academy of Science and Technology. E-mail: ptgiang@ioit.ac.vn

() The Authors. Published by Blue Eyes Intelligence Engineering and Sciences Publication (BEIESP). This is an open access article under the CC BY-NC-ND license (http://creativecommons.org/licenses/by-nc-nd/4.0/) testbed system. One of the typical testbed is ORBIT[2, 3].

There are many studies evaluating wireless network performance using testbed. Study[4] setup a simple testbed consisting of two machines acting as client/server that communicates via an Access Point to send and receive two types of TCP/UDP data in two cases: using the Enhanced Distributed Channel Access - EDCA and use the traditional Distributed Coordination Function - DCF. When using the lower value of Signal-to-Noise Ratio (SNR) by increasing the distance between the AP and the receiver, the test results show that TCP throughput is greatly affected by lost frames due to low SNR values.

Research[5] assess the performance of IEEE 802.11 DCF networks by using testbed to simulate environment with a large number of users as well as high density AP, and focus on key DCF parameters such as initial contention window size $\left(C W_{\min }\right)$ and maximum contention window size $\left(C W_{\max }\right)$ to evaluate their impact on network performance, the evaluation results are also compared to an analytics model and the result shown that by changing $C W_{\min }$ according to WiFi offered load, maximum throughput of network can be achieved and optimal $C W_{\min }$ value will change as the network size changes.

The paper[6] illustrates the development of a testbed system for multi-hop mobile wireless mesh networks that plays an important role in modern technologies such as Smart-Cities or Internet-of-Things. Testbed was built on open source software like Linux and low-cost computers like the Raspberry Pi and evaluates the network in a variety of test cases to show that the variables are not controlled like the number of stages of the network nodes, the path of the data, the number of data flows has a great impact on system performance.

\section{RESEARCH METHODOLOGY}

As analyzed above, we see that the testbed-based method has many advantages compared to the modeling and simulation methods. Therefore, in this paper we will use a testbed system to evaluate the QoS parameters. In our paper, we present some evaluation experiments of wireless network performance for multimedia data. With this type of data, modeling[7] and simulation[8] methods can be used because it is based on model analysis as well as simulation code in accordance with the IEEE 802.11e[9], which is a standard for multimedia data. However, due to the fact that the data for this type of data are now included in the popular IEEE 802.11a,b,g,n[1] standards, we set up a testbed model using the above standards, for example IEEE $802.11 \mathrm{~g}$ to conduct the emulation. 


\section{EVALUATE QOS PARAMETERS OF MULTIMEDIA DATA TYPES}

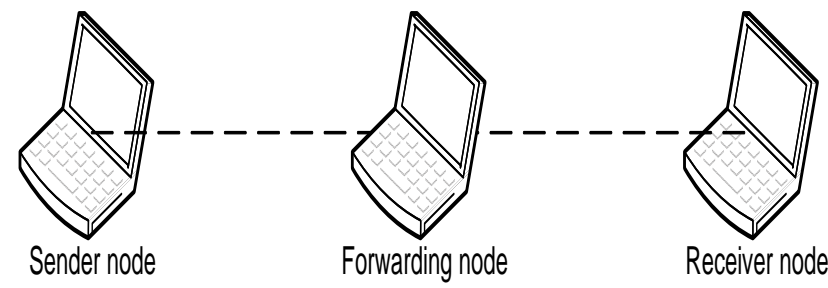

Fig. 1.Multi-hop wireless ad hoc network

Figure 1 is a testbed included two connected wireless nodes in ad hoc mode via an forwarding node, these three nodes support QoS for multimedia data (WiFi Multimedia or WMM feature) with IEEE 802.11g. This ad hoc topology is setup by hostapd[10] with WMM turned on. The default QoS parameters of IEEE 802.11g, with four Access Category types with priority levels: AC_BK (background), AC_BE (best effort), AC_VI (video) and AC_VO (voice) with corresponding IEEE 802.11 QoS[11] parameters set from lowest to highest, are shown in Table I:

Table- I: IEEE 802.11 QoS parameter set

\begin{tabular}{|c|c|c|c|c|}
\hline AC & CWmin & CWmax & AIFSN & TXOP limit \\
\hline AC_BK & 15 & 1023 & 7 & 0 \\
\hline AC_BE & 15 & 1023 & 3 & 0 \\
\hline AC_VI & 7 & 15 & 2 & 3008 \\
\hline AC_VO & 3 & 7 & 2 & 1504 \\
\hline
\end{tabular}

To evaluate the sending and receiving processes between Sender/Receiver nodes in Figure 1, we use iPerf [12], a network performance testing software that generates TCP and UDP data, as well as allows changing parameters such as bandwidth, TCP/UPD packet size, number of packets sent/received, bidirectional connection, window size (with TCP data), etc.

In order to evaluate the priorities between Voice, Video and Background data with IEEE 802.11g, we perform experiments by sending simultaneously three data flows at saturation state in 60 seconds, and we repeated the experiment 10 (ten) times to get average values, so that there are no significant variants in stable data.

First of all, we conducted the above experiments to compare the difference between the two mechanisms: $D C F$ (default in 802.11) and EDCA (QoS support with multimedia data in 802.11). The results are as shown in Tables II and III.

Table- II: Experiment result of DCF mode

\begin{tabular}{|c|c|c|c|}
\hline Data type & $\begin{array}{c}\text { Throughput } \\
(\mathrm{Mbps})\end{array}$ & $\begin{array}{c}\text { Jitter } \\
(\mathrm{ms})\end{array}$ & $\begin{array}{c}\text { Loss ratio } \\
(\%)\end{array}$ \\
\hline Voice & 3.16 & 41.21 & 0 \\
\hline Video & 3.15 & 32.39 & 0 \\
\hline Background & 3.15 & 32.62 & 0 \\
\hline
\end{tabular}

Table-III: Experiment result of EDCA mode

\begin{tabular}{|c|c|c|c|}
\hline Data type & $\begin{array}{c}\text { Throughput } \\
(\mathrm{Mbps})\end{array}$ & Jitter (ms) & $\begin{array}{c}\text { Loss ratio } \\
(\%)\end{array}$ \\
\hline Voice & 8.47 & 13.59 & 0.03 \\
\hline Video & 2.07 & 26.74 & 9.99 \\
\hline Background & 0.11 & 1667.40 & 92.4 \\
\hline
\end{tabular}

Look at the performance results of DCF and EDCA when the network is at saturation state. We see that because DCF does not distinguish the priority of the data types, the performance of the three data types is quite similar in all three value throughput, jitter and packet loss.

With EDCA, priority level makes a clearly impact on network performance. Obviously, the throughput of the background data (the lowest priority) is almost zero corresponding to the large packet loss rate (complete loss), as well as large jitter value indicates unstable data transmission. While the higher-priority data take up most of the bandwidth, as well as the relatively small jitter and packet loss values, representing a stable data transmission, suitable for multimedia data.

We continue to evaluate the effect of changing $C W_{\text {min }}, A I F S$, and $T X O P_{\text {limit }}$ values to the throughput of each data type. To do so, first we set all parameters with the same value as Table IV.

Table- IV: The same value of WMM parameters

\begin{tabular}{|c|c|c|c|c|}
\hline AC & CWmin & CWmax & AIFSN & TXOP limit \\
\hline AC_BK & 7 & 1023 & 7 & 0 \\
\hline AC_BE & 7 & 1023 & 7 & 0 \\
\hline AC_VI & 7 & 1023 & 7 & 0 \\
\hline AC_VO & 7 & 1023 & 7 & 0 \\
\hline
\end{tabular}

Looking at the experiment results with above parameters set in Table V. It is easy to understand that Table $\mathrm{V}$ will give similar results to Table II, but the results are a bit worse (larger jitter and packet loss ratio, smaller throughput). This is because the QoS parameter set has a certain effect on network performance, even if all the parameters are equal.

Table- V: Experiment result of EDCA mode with the same QoS parameters

\begin{tabular}{|c|c|c|c|}
\hline Data type & $\begin{array}{c}\text { Throughput } \\
(\mathrm{Mbps})\end{array}$ & $\begin{array}{c}\text { Jitter } \\
(\mathrm{ms})\end{array}$ & $\begin{array}{c}\text { Loss ratio } \\
(\%)\end{array}$ \\
\hline Voice & 3.08 & 36.71 & 0.19 \\
\hline Video & 3.07 & 39.02 & 0.18 \\
\hline Background & 3.09 & 37.08 & 0.16 \\
\hline
\end{tabular}

\section{A. Validation of Contention Window (CW)}

Next, we demonstrate the relationship between Contention Window size of three data types (Voice, Video, and Background). To do that, we fixed two parameter sets of the highest (voice data) and the lowest (background data) priorities and step-by-step changing $\mathrm{CW}$-size of video data. The range of $\mathrm{CW}$ values of three types of data which also change from arcording to Table I and $\mathrm{CW}_{\text {min }}$ size of the video data which will change in that range ( 3 to 15 ) to give an observation of how the throughput ratio of three data types changes corresponding to $\mathrm{CW}$ changes.

Looking at the experiment result in Figure 2, 3, and 4, we see that the $\mathrm{CW}_{\text {min }}$ size greatly affects network throughput, small $\mathrm{CW}$ has large throughput, and vice versa. With default $\mathrm{CW}_{\text {min }}$ (7), the video throughput is still as large as the default priority. But when the $\mathrm{CW}_{\text {min }}$ increases larger, the throughput of video data immediately dropped very quickly, as well as jitter and packet loss ratio become much worse.

Published By:

Blue Eyes Intelligence Engineering \& Sciences Publication 


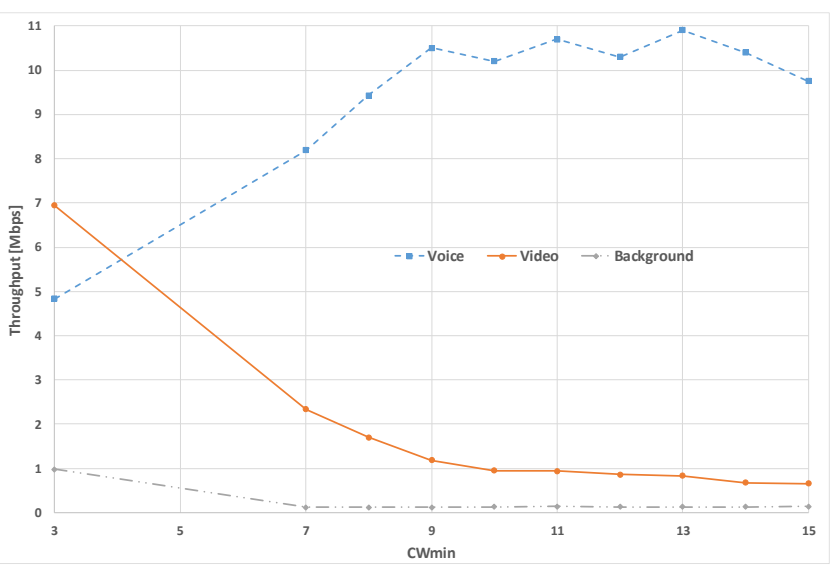

Fig. 2. Throughput comparison by CWmin

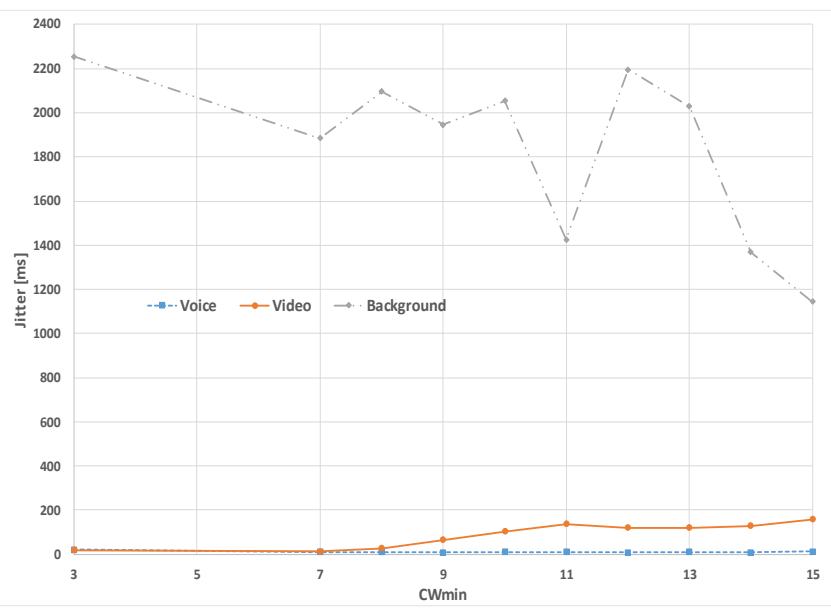

Fig. 3.Jitter comparison by CWmin

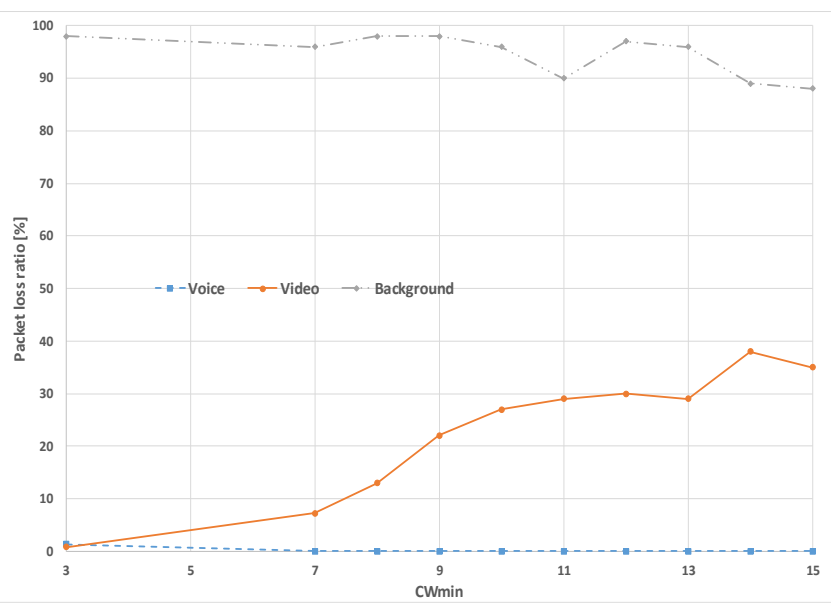

Fig. 4.Packet loss comparison by CWmin

\section{B. Validation of TXOP}

The effect of Transmission Opportunity (TXOP) limit is simple. If this value gets larger, the throughput of corresponding node becomes larger, too. Otherwise, the jitter (or delay in average) for other nodes would be increased because these nodes must wait for a longer transmission time to send their packets. Because of the largest TXOPlimit value (3008 microseconds) as default in IEEE 802.11. We observe the change of network performance by changing the TXOP values of voice instead of video data. As shown in Figure 5, if the TXOP value of voice data gets larger, the corresponding throughput becomes larger. But when the TXOP of voice increases to its deafault value (47 in Figure 5 or 1504 microseconds in Table I), the throughput is still

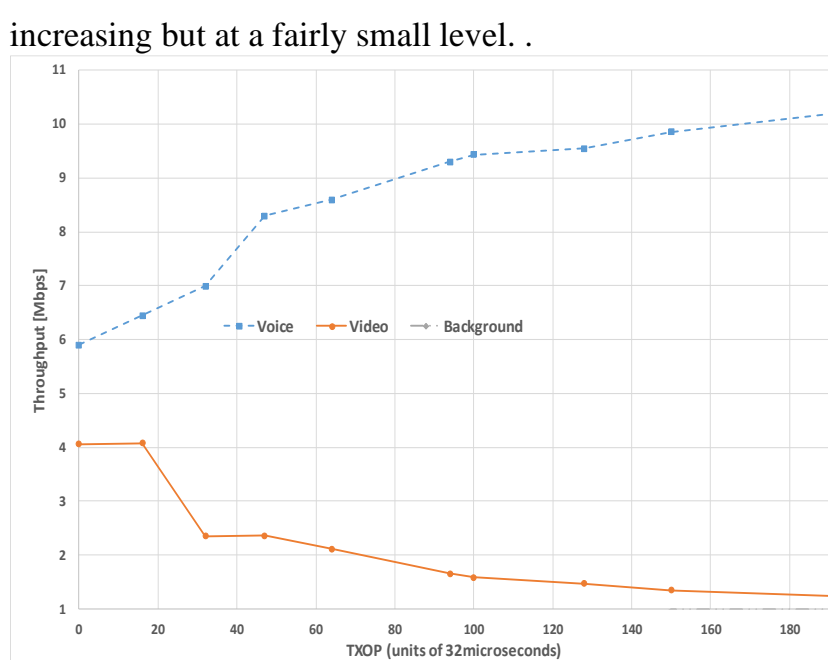

Fig. 5.Throughput comparison by TXOPlimit

Figures 6 and 7 indicate that TXOP does not affect the jitter and packet loss ratio values as much as throughput. The variation level of the delay (latency) of the voice data is still smaller (better) than the video, and the packet loss ratio of the video is still greater (worse) than the voice. And background data always has a much worse performance than the other two types of data.

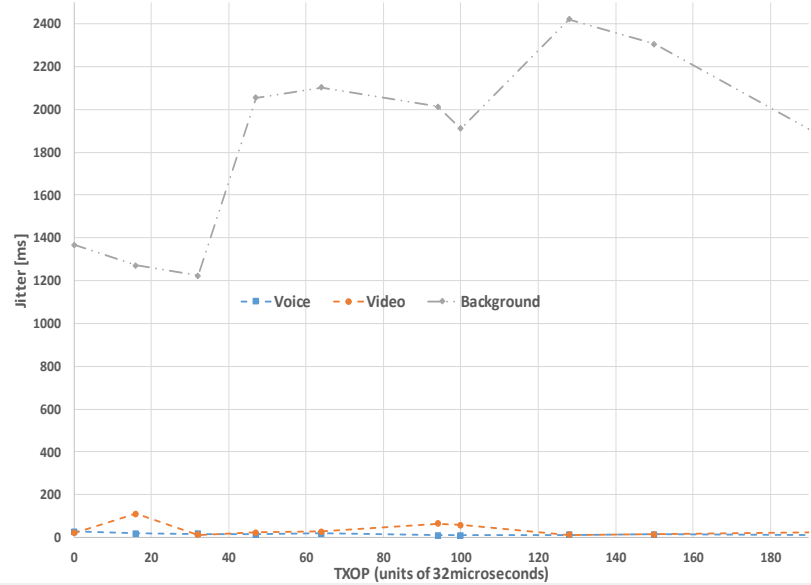

Fig. 6.Jitter comparison by TXOPlimit

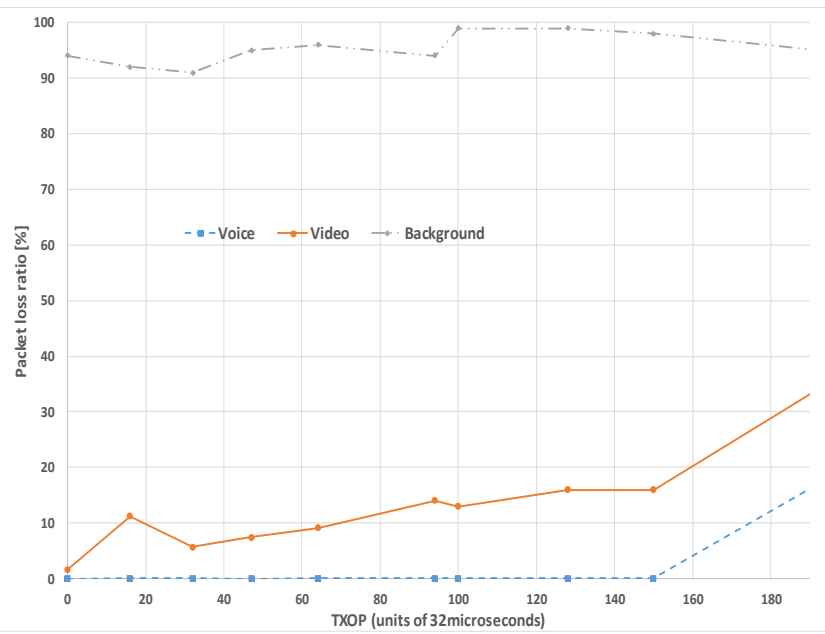

Fig. 7.Packet loss comparison by TXOPlimit

Published By:

Blue Eyes Intelligence Engineering \& Sciences Publication 


\section{Validation of AIFS}

The effect of AIFS number is shown in Figures 8, 9 and 10. Different AIFSN values are used for different types of data (AC), so higher priority node will use smaller AIFSN values, this smaller AIFSN value will cause the station to wait a duaration less time before the media can be accessed or the back-off countdown starts up than the lower priority node. Therefore, higher priority nodes are guaranteed to be able to use higher bandwidth than lower priority nodes.

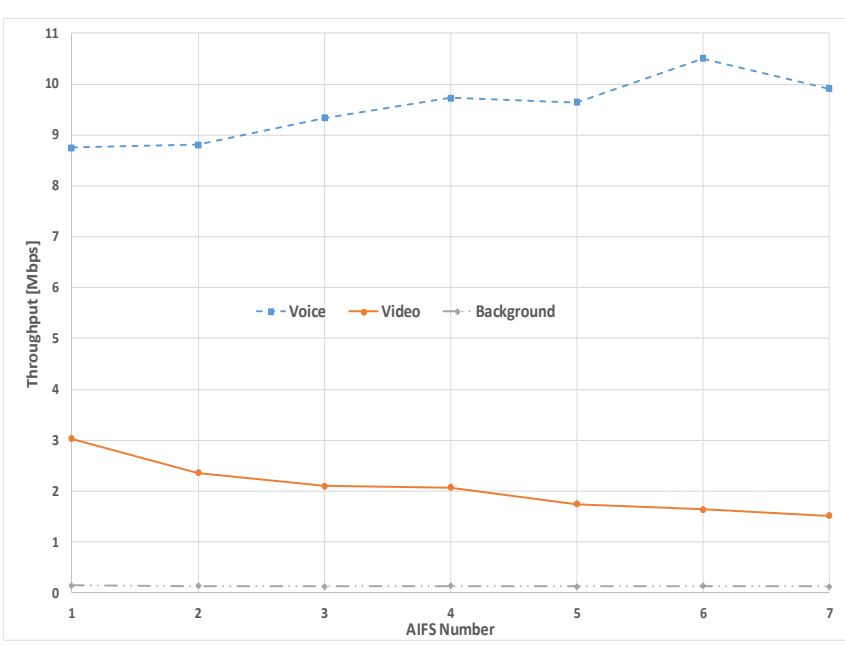

Fig. 8.Throughput comparison by AIFS Number

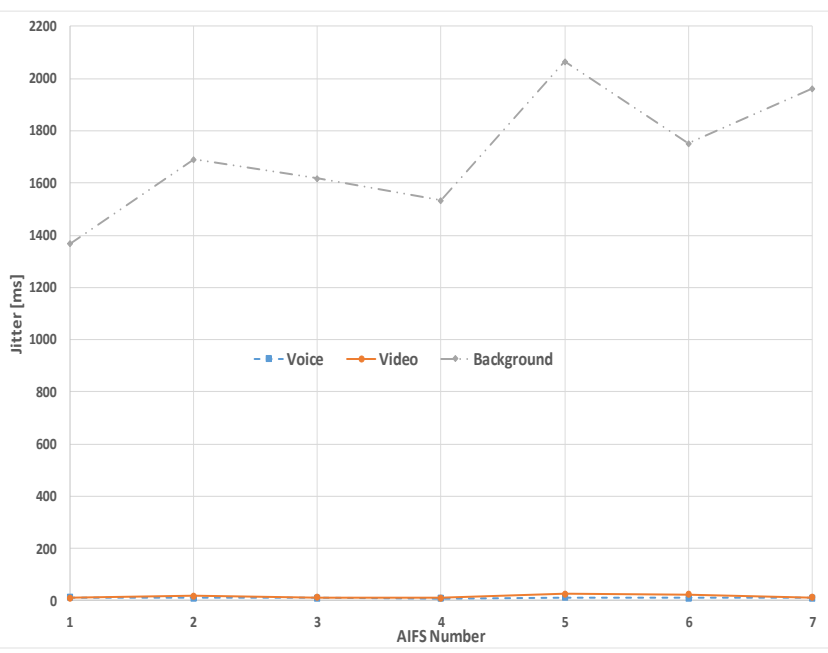

Fig. 9.Jitter comparison by AIFS Number

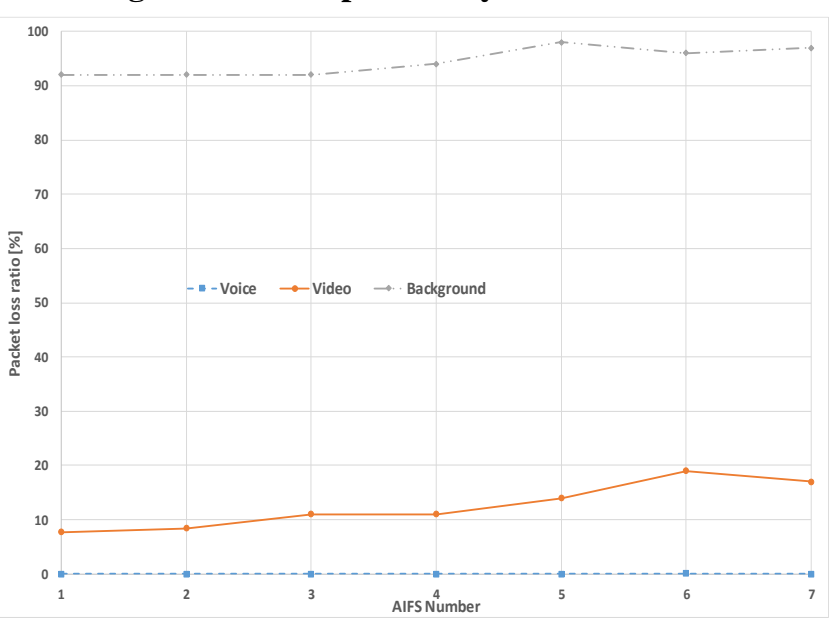

Fig. 10. Packet loss comparison by AIFS Number

\section{The effect of QoS parameters on performance}

After evaluating the effects of $C W_{\min }, T X O P$ and AIFS above. We can realize that the $C_{\text {min }}$ size has a great influence on the throughput of multimedia data. Obviously, just a small change of $\mathrm{CW}_{\min }$ makes the throughput increase or decrease very quickly. While TXOP and AIFS values also make changes but are minor. In multimedia data networks, throughput is an important factor affecting network performance. Thus, it can be seen that if we want to adjust the throughput ratio between multimedia data types, we can control it via $\mathrm{CW}_{\min }$ value.

\section{CONCLUSION}

Wireless technology is becoming an important infrastructure of small and medium, even industrial scope. However, technologies used in wireless networks could be evaluated and verified or tested many times before they are published for official use. Many studies of wireless networking technologies were based on mathematical models, or simulation tools. These methods have the advantage of no-expensive hardware because they are primarily mathematical proofs or use software tools to write simulation scripts, analyze results, ... and limited by idealistic conditions and assumptions. Besides, modeling or simulation cannot fully reflect the physical components of the network. The trend of using testbed to evaluate network parameters is increasingly, and showing superiority compared to modeling and simulation methods. Therefore, this research has focused on building a network testbed with reference to the existing testbeds already available in the world. Therefore, we are successfully deploying the testbed and evaluating some QoS parameters for multimedia data in IEEE 802.11 wireless network.

\section{ACKNOWLEDGMENT}

This research was funded by the Institute of Information Technolog (IOIT), Vietnam Academy of Science and Technology (VAST).

\section{REFERENCES}

1. "IEEE 802.11 Working Group Project Timelines," http://grouper.ieee.org/groups/802/11/Reports/802.11 Timelines.htm, last accessed on 02/12/18.

2. M. Ott, I. Seskar, R. Siraccusa, and M. Singh, "Orbit testbed software architecture: supporting experiments as a service," in First International Conference on Testbeds and Research Infrastructures for the DEvelopment of NeTworks and COMmunities, Feb 2005, pp. 136-145.

3. D. Raychaudhuri, I. Seskar, M. Ott, S. Ganu, K. Ramachandran, H Kremo, R. Siracusa, H. Liu, and M. Singh, "Overview of the ORBIT radio grid testbed for evaluation of next-generation wireless network protocols," in IEEE Wireless Communications and Networking Conference, 2005, vol. 3, March 2005, pp. 1664-1669 Vol. 3.

4. S. Mukherjee, X. Peng, and Q. Gao, "QoS Performances of IEEE 802.11 EDCA and DCF: A Testbed Approach," in 2009 5th International Conference on Wireless Communications, Networking and Mobile Computing, Sep. 2009, pp. 1-5. 
5. E. Z. Chen, D. Fu, Y. Gao, and X. Hei, "Performance Evaluation for WiFi DCF Networks from Theory to Testbed," in 2017 IEEE International Symposium on Parallel and Distributed Processing with Applications and 2017 IEEE International Conference on Ubiquitous Computing and Communications (ISPA/IUCC), Dec 2017, pp. 1364-1371.

6. P. A. Regis, C. Miley, and S. Sengupta, "Multi-hop mobile wireless mesh network testbed development and measurements," International Journal of Innovative Research in Computerand Communication Engineering, vol. 5, no. 8, August 2017.

7. I. Tinnirello and G. Bianchi, "Rethinking the IEEE 802.11e EDCA Performance Modeling Methodology," IEEE/ACM Transactions on Networking, vol. 18, no. 2, pp. 540-553, April 2010.

8. N. H. Anh and P. T. Giang, "An Enhanced MAC-Layer Improving to Support QoS for Multimedia Data in Wireless Networks," Indian Journal of Science and Technology, vol. 9, no. 20, 2016. [Online]. Available: http://www.indjst.org/index.php/indjst/article/view/92732

9. “IEEE 802.11e Amendment," https://standards.ieee.org/standard/802 11e-2005.html, last accessed on 12/11/18

10. "hostapd: $\quad$ IEEE $802.11 \quad$ AP, IEEE 802.1X/WPA/WPA2/EAP/RADIUS Authenticator," https://standards.ieee.org/standard/802 11-1999.html, last accessed on 30/09/19.Book

11. “IEEE 802.11-2012,” https://standards.ieee.org/standard/802 11-2012.html, last accessed on 02/12/18.

12. "iPerf - The ultimate speed test tool for TCP, UDP and SCTP," https://iperf.fr/, last accessed on 03/12/19.

\section{AUTHORS PROFILE}

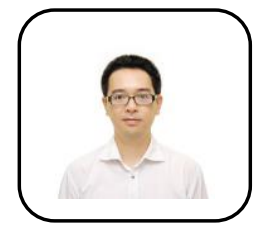

Ngo Hai Anh, received the M.Tech degrees from Vietnam National University and is $\mathrm{PhD}$ student at Graduate Univeristy of Science and Technology, Vietnam Academy of Science and Technology. He is currently an researcher at Telemactis Department of Institute of Information Technology, Vietnam Academy of Science and Technology. His current research interests include wireless network, network performance and network management.

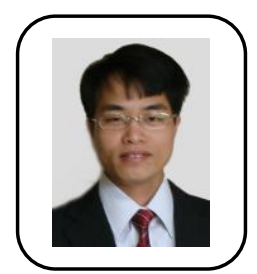

Pham Thanh Giang, received the B.E. degree from the Hanoi University of Technology, Vietnam, in 2002. He achieved M.E. and D.E. degrees from the Nagaoka University of Technology, Japan, in 2007 and 2010, respectively. He is currently a researcher at Institute of Information Technology, Vietnam Academy of Science and Technology. His interests are security, mobile ad hoc networks, the Internet traffic measurement architecture in mobile environments and Internet 